\title{
Material objects and essential bundle theory
}

\author{
Stephen Barker ${ }^{1}$ (D) Mark Jago ${ }^{1}$
}

(C) The Author(s) 2017. This article is an open access publication

\begin{abstract}
In this paper we present a new metaphysical theory of material objects. On our theory, objects are bundles of property instances, where those properties give the nature or essence of that object. We call the theory essential bundle theory. Property possession is not analysed as bundle-membership, as in traditional bundle theories, since accidental properties are not included in the object's bundle. We have a different story to tell about accidental property possession. This move reaps many benefits. Essential bundle theory delivers a simple theory of the essential properties of material objects; an explanation of how object coincidence can arise; an actual-world ground for modal differences between coincident objects; a simple story about intrinsic properties; and a plausible account of certain ubiquitous cases of causal overdetermination.
\end{abstract}

Keywords Ontology · Material objects · Bundle theory $\cdot$ Essence $\cdot$ Nature

\section{Introduction}

How should a metaphysician characterise material objects? Are they mere masses of matter? Bundles of properties? Regions of spacetime? Substrata? Traditional attempts to answer these questions diverge on whether there is something to being a given object, aside from its properties. According to bundle theories, material objects are 'bundles' of their qualities. There is nothing to being the object, other

Mark Jago

mark.jago@nottingham.ac.uk

Stephen Barker

stephen.barker@nottingham.ac.uk

1 Department of Philosophy, University of Nottingham, University Park, Nottingham NG7 2RD, UK 
than its properties, bundled together. An alternative (broadly Kantian) approach is to take a material object to be its properties, insofar as they are thought about in a certain combining manner' (Bennett 1987: 202). Other views hold that there must be some aspect of the object that remains constant through change, providing the identity of the continuing object. These are substance theories, and their task is to articulate the nature of this extra ingredient.

Our aim in this paper it to introduce a new answer to the question, what are material objects?, which falls somewhere between these traditional answers. It incorporates elements of both bundle and substance theories, together with new ideas which, we think, add up to a new approach. On our final theory, objects are defined in terms of property instances, in a way that echoes bundle theory. (We'll say what we mean by 'property instance' in Sect. 2). But our understanding of those property instances requires a substance-like notion, which, for us, is spacetime. Reality is fundamentally a totality of concrete spacetime and its property instances. Material objects are metaphysically derivative entities. (Perhaps fundamental reality includes physical laws too. We're open to this possibility, but that discussion is beyond the scope of this paper. As far as we can see, the theory we develop here is compatible with any of the main philosophical views of laws).

Our theory includes two standout features. First, and most importantly, when we identify a material object with a bundle of property instances, we understand those properties as telling us about the nature or essence of the object in question. (We explain our reasons in Sect. 3. Throughout, we'll use 'nature' and 'essence' as synonyms). We do not analyse $x$ 's possessing $F$ in terms of the $x$-bundle containing (an instance of) $F$, as traditional bundle theories do. We formulate distinct (nonbundle membership) conditions for property possession (Sect. 5). This component of our theory makes it (as far as we know) completely unique.

Second, we allow for there to be distinct yet permanently coincident material objects, differing from one another in their natures. This feature arises naturally from our account of when property instances form a material-object-constituting bundle (Sect. 2). We take the feature to be a benefit of our account, for reasons we discuss in Sect. 4.

A new and unusual theory needs care in its articulation. We'll spend most of our time here doing just that. We won't criticise alternative theories in any detail, or seek to demonstrate how our proposed theory fares in comparison. We'll indicate how our approach addresses a range of issues we find with other theories. But we leave it up to the reader to judge how other theories fare on those issues.

What are the criteria for a good metaphysical theory of material objects? There are many, but we think the following mixed bag are all important:

(1) It should tell us something about the metaphysical nature of an object (or else be in a position to explain away talk of objects having natures at all).

(2) It should allow for (or at least explain the appearance of) distinct but indiscernible material objects.

(3) It should explain cases in which we appear to have distinct yet permanently coincident objects. 
Each of these criteria has been the focus of a great deal of metaphysical discussion. In each case, we should allow the theoretical possibility that there's no good answer to be had. But in that case, the theory of objects should explain why we're misled into asking for a metaphysical answer.

There are further criteria which, whilst perhaps not essential to a theory of material objects, nevertheless are important to our understanding of what material objects are:

(4) We'd like an account which explains how material objects possess properties, and stand in relations to one another.

(5) We'd like an account of whether a property had by an object is intrinsic to that object.

(6) We'd like a story about cases of (seeming) causal overdetermination.

We think our approach provides us with a good explanation in each case.

\section{Bundles and substances}

Our theory is built on a foundation of properties-in-spacetime. These combine (under certain conditions) to give us material objects. In this section, we'll explain what we mean by this, and why we think a theory of material objects needs both of these components.

We take as our fundamental ontological categories property and spacetime. We'd be equally happy to work with some other kind of space-configuration space, phase space, or whatever-should it turn out that spacetime really isn't fundamental. (We think that's a live option. We defer to whatever turns out to be the best philosophical interpretation of whatever turns out to be the best fundamental physics. So treat our talk of 'spacetime' as something of a placeholder).

We take our properties to be broadly of the Platonic variety: universals. But nothing much hangs on this. If you prefer some other theory of properties, substitute it throughout. All we require is that properties exist and are not metaphysically dependent, for their identity or existence, on the objects that possess them.

Our picture of fundamental reality is of fundamental properties (which might include mass, spin, and charge) distributed across spacetime (or perhaps some other kind of space). We're happy to defer to fundamental physics on the question of the fundamental properties. If mass or charge are best explained by a theory which takes other properties to be more basic, then we'll take those other properties to be more fundamental than mass or charge. If fundamental physics is best formulated in terms of configuration space (say), rather than directly in terms of spacetime, then we'll take configuration space to be our fundamental space (Arntzenius 2014).

Each way of distributing the fundamental properties gives a different possible world. A good deal of work in our theory is done by the resulting properties-inspacetime, which we'll call property instances. These are specific located instances of charge, or mass, or redness, or penguinhood (in contrast to a multi-located property-type or universal). The charge of this electron, here, or the redness of that postbox, there, are instances of the universal properties charge and redness. 
Metaphysically, we think of a property instance as involving a property (universal) and a region of spacetime. Just how universal and spacetime region combine to form a property instance is up for grabs. We might conceptualise the 'metaphysical glue' in question along the lines of Armstrong's (2010) 'fundamental tie' (although, in our case, the resulting entity is a property instance, and not one of Armstrong's state of affairs). But one could instead understand property instances along the lines of primitive tropes (Stout 1921/1971; Williams 1953), which are not composed of further entities. All that matters for our theory is that each property instance can be associated with a unique universal and a unique spatiotemporal region (whether or not these compose that property instance). We'll say that the (universal) property is instanced throughout that region, and that that region instantiates the property.

One might use this approach to give a substance-based theory, as Schaffer (2009), Skow (2005), and Sider (2003) do, by identifying material objects with regions of spacetime. (This approach is known as supersubstantivalism). We have worries about that approach. We think that artworks, coins, utterances, and people are material objects which have various kinds of value to us. But the spatiotemporal regions they occupy don't possess those values. Most material objects could have been elsewhere right now, could have been larger or smaller, and could have been brought into existence earlier or later than they in fact were. Not so for the spatiotemporal regions they inhabit, for they are defined in terms of their locations, sizes, and durations.

That, in short, is why we don't want to identify material objects with the spatiotemporal regions they inhabit. Those who think otherwise have their responses. They may accept a version of counterpart theory on which ' $a$ might have been $F$ ' and ' $b$ could not have been $F$ ' do not jointly imply that $a$ and $b$ are distinct. (That helps with the modal cases. The non-modal cases must be explained some other way). We have argued elsewhere against any such approach (Barker and Jago 2014).

The identity of a material object is not (just) about the where and when. A material object is a person, or a table, or a sculpture-in general, some combination of qualities - taking a journey through space and time. That is our guiding idea in identifying material objects with 'bundles' of property instances. The (universal) properties involved in those instances give us the nature of the object-person, table, or sculpture-and the spatiotemporal region of those instances is the object's path through spacetime. This is the impressionistic canvas which we aim to transform into a precise portrait.

First of all, we owe a story about what it is for property instances to 'bundle' together. Any such account faces three immediate difficulties. First, bundle theories seem committed to the questionable principle of the identity of indiscernibles. Second, why do these properties, but not those, comprise an object? And third, objects change their properties over time (and across worlds), whereas bundles seem unable to. 
Commitment to the identity of indiscernibles is often taken as a knock-down objection to bundle theory. We agree that the principle is deeply unwelcome: there may be distinct but qualitatively identical entities (as in Black's (1952) symmetrical universe). But the issue does not arise if we work with our notion of a property instance. Each such instance is located in the associated region of spacetime (and indeed, it's intrinsic to that instance that it's located in that region). As a consequence, spatiotemporal differences (even in a symmetrical universe) suffice for distinctness. Our approach instead entails the numerical identity of permanently co-located, qualitatively identical entities. We're much happier with that principle.

One traditional answer to the 'bundling' issue is: properties bundle together when they are co-located. This answer is hard to articulate fully on a traditional bundle theory, for how are we to understand the location of a property, in and of itself? (Hawthorne and Sider (2002) discuss the options). Our approach does not face this problem, as each property instance has its location intrinsically. Nevertheless, we do not accept the 'co-location' answer, for it entails a principle we reject: that colocation (of material objects) implies identity. The sculpture Continuum could have been constituted from some other sheets of bronze. The co-located sheets of bronze from which it is made might have constituted nothing of value. It's the sculpture, not the material from which it's made, that's (artistically and financially) valuable. It's for precisely that reason that artworks are renovated, sometimes by replacing bits of their matter. Our reasons against identifying a material object with a spacetime region (Barker and Jago 2014) apply just as strongly against identifying (even permanently) coincident material objects.

As a consequence, we do not define 'bundling' of property instances in terms of their location. We allow co-located property instances to be parts of distinct (and colocated) bundles. An instance of being an artwork will be in the Continuum-bundle, but not in the mass-of-bronze-bundle, for example. So, for any spatiotemporal region, the property instances instantiated in it may bundle together in distinct ways, constituting distinct material objects.

Nevertheless, co-location is necessary for property instances to bundle together to constitute a material object, in the following sense:

(Unification) Property instances constitute a material object only if there is a spatiotemporal region $r$ such that each such property instance has $r$ as its region.

Each property instance is associated with a unique spatiotemporal region, throughout which it is instantiated. Note that we said 'throughout', not 'in'. Being a sculpture is instanced throughout the twisting region of spacetime occupied by Continuum. That region is subsumed by the US's spatiotemporal region. But we mustn't infer from this that being a sculpture is instanced throughout the US's region. Each property instance is associated with exactly one region. Property instances constitute a material object only when they are all associated with precisely the same region.

(Given further assumptions, which we discuss below, the converse of UNIFICATION may also hold: any co-located property instances bundled together constitute a 
material object. This is consistent with distinct but co-located material objects. What we deny is that there is but one bundle for any given spatiotemporal region, consisting of all the property instances instantiates throughout that region).

Note that UNIFICATION still allows a very liberal notion of a material object, as we have not insisted that spatiotemporal regions must be spatially or temporally continuous. We do not rule out spatially discontinuous objects, such as the Australian Capital Territory, and temporally discontinuous objects, such as the person who, due to patters of conscious continuity, exists only on Mondays, Wednesdays and Fridays (Braddon-Mitchell and West 2007).

We also want bundles to be logically consistent and closed, in the following senses:

(CONSISTENCY) For any property $F$, no bundle of property instances contains both $F$ and non-Fness.

(Closure) Every bundle of property instances is truthmaker-closed.

To understand this latter notion, say that a proposition $\langle A\rangle$ truthmaker entails a proposition $\langle B\rangle$ just in case any possible truthmaker for $\langle A\rangle$ is also a truthmaker for $\langle B\rangle$. Then a bundle $b$ of property instances is truthmaker closed just in case: if the conjunction $\left\langle F_{1} x \wedge F_{2} x \wedge \cdots\right\rangle$ truthmaker entails $\langle G x\rangle$ (for arbitrary $x$ ) and $b$ contains instances of each of $F_{1}, F_{2}, \ldots$, then $b$ contains an instance of $G$. (We include the cases in which the premise is an infinite conjunction and in which it is a single conjunct, $\langle F x\rangle$ ). Intuitively, the condition guarantees that, when some property instances in a bundle ground a further property instance, then that further instance is also in the bundle. Precise details of the logic of truthmaker entailment can be found in (Fine and Jago 2017).

The closure condition ensures that bundles containing, say, is red will also contain is coloured. If there are disjunctive properties, then bundles containing is red will also contain is red or blue. In general, bundles contain a determinable property whenever they include one of its determinates. But note that bundles are not required to be downwards-closed: a bundle may contain a determinable without containing any of its determinates. This will be important for the use to which we put bundles in Sect. 3.

We take these three necessary conditions on bundling-UNIFICATION, CONSISTENCY, and CLOSURE- to be jointly sufficient. Pluralities of property instances that meet all three conditions thereby constitute a material object.

What is a bundle, metaphysically speaking? How do we get from a diverse plurality of property instances to a unified entity? Following McDaniel (2001), Paul (2002, 2006), and Williams (1953), we take bundles to be mereological sums (fusions) of properties (or of their instances, in our case). Mereological summation delivers unity from plurality (just as set formation does): the sum of some $x s$ is the least entity (with respect to parthood) which overlaps everything the $x s$ overlap. This is precisely what we want.

One might take our three conditions to be conditions on when mereological summation (of property instances) occurs. Alternatively, if you think that any plurality forms a mereological whole (as Lewis (1986), Heller (1990), and Sider (2003) do), then take our three conditions as conditions on which mereological sums 
of property instances count as material objects. (If we accept that line, then UNIFICATION becomes a biconditional, whilst still allowing coincident bundles). We prefer this latter option, but little hangs on this. Indeed, not much hangs on our mereological treatment of bundles. If you prefer some other way of getting unity from plurality (or are happy to think of each material object as a plurality) then you're welcome to understand our 'bundle' talk in those terms.

We thus arrive at a picture of what material objects are:

(Material OBJECT) A material object is a consistent and truthmaker closed mereological sum of property instances, all of which are instanced throughout precisely the same spatiotemporal region.

\section{The nature thesis}

Material objects are bundles: mereological sums of property instances meeting our three conditions. So we say. But a bundle is the bundle it is because of what's thereby bundled. The property instances constituting the bundle provide its identity. Remove one property instance, and you have a different bundle. Not so for material objects, which typically could have been many different ways. So how can we identify a material object with a bundle?

We take this objection seriously. We think it seriously damages traditional bundle theories. (Paul (2006) considers some responses, which we discuss in Sect. 4; see also (Jago 2016)). But ours is a bundle theory with a twist. Having identified object with bundle, we interpret that bundle as saying what the object is. The properties in the bundle give us the nature, or essence, of the material entity. This is essential bundle theory. In this section, we set out what this means, and why we think it's a good move to make.

Traditional bundle theories encapsulate two key theses about the role of bundles:

(Constitution Role) Material objects are identified with bundles of properties and relations (or their instances), so that the bundled properties and relations (or their instances) constitute material objects.

(Property POSSESSION ROLE) An object $x$ possesses property $F$ if and only if $F$ is a member of the $x$-bundle.

Essential bundle theory accepts CONSTITUTION ROLE for bundles, but rejects PROPERTY POSSESSION ROLE. It is not the case, on our theory, that an object $x$ is $F$ just in case $F$ is in the $x$-bundle.

What reason do we have for rejecting PROPERTY POSSESSION ROLE? A good answer to the question, 'what is that object?' need not be an answer to 'how is that object?', i.e., what properties does it have? Typically (outside the philosophy classroom) we answer 'what is that?' by reference to a sortal: we say 'it's a jumper' or 'it's a statue' or 'it's so-and-so's latest song'. In giving these answers, we're singling out the object via its nature or essence (Wiggins 1980). If you're tempted by that way of thinking (as we are), then it is rather awkward to align the object constitution role with the property possession role. Object constitution is a matter of what the object 
is and, on the view we're considering, what the object is amounts to the object's nature or essence. How the object is-it's full qualitative profile-is another matter. We don't say that one must think in these terms. But if one does think in these terms, then one has a good reason for pulling apart the object constitution and the property possession roles: bundles play the former, but not the latter.

We reject PROPERTY POSSESSION ROLE and, in its place, adopt the following:

(Nature thesis) Material object $x$ is essentially $F$ if and only if an $F$-instance is part of the $x$-bundle.

On this picture, bundles of property instances give the nature of the object in question. When an instance of $F$ is in a bundle $x$, that object is essentially $F$. Fness is, quite literally, part of what $x$ is, and so is part of $x$ 's nature. The resulting theory of material objects, which accepts CONSTITUTION ROLE and NATURE THESIS but rejects PROPERTY POSSESSION ROLE, is essential bundle theory.

The resulting theory is what Fine (1995) calls a constitutive (in contrast to a consequential) notion of nature or essence. The former, but not the latter, 'is directly definitive of the object' (Fine 1995: 57). On this approach, the nature of an object reveals a deep fact about an object. This is in contrast to the Lewisian counterparttheoretic view (Lewis 1986), on which essence-talk is ultimately analysed in terms of similarity. On that story, for (just about) any property $F$ and any object $x$ that's $F$, there will be a context in which $x$ is essentially $F$ (Lewis 2003; Lewis and Rosen 2003). Truths about essence then seem very cheap indeed. Our approach also provides a more nuanced, fine-grained approach to essence than counterpart theory. It's de re necessary of Bill that $2+2=4$, but this is no part of his nature. Lewisians cannot capture this fact, since they align essence-talk with de re necessity.

We can also contrast essential bundle theory with the 'nuclear theory' of Simons 1994. Simons uses a bundle of tropes, which he calls the 'essential kernel' and 'nucleus' of the object, to give that object's nature or essential properties (Simons 1994: 567). In this respect, the theories are similar; but the similarity ends there. Simons thinks of a bundle-of-tropes 'nucleus' as an object's substratum, upon which the object's accidental tropes depend. Those accidental tropes form a 'looser' bundle around the 'tight' substratum bundle (Simons 1994: 568). So Simons analyses both essential and accidental property possession in terms of bundlemembership, with one 'tight' bundle giving the object's essential properties and a further 'loose' bundle giving the object's accidental properties.

This approach suffers from the objection we raised above. If a bundle's identity is fixed by the properties thereby bundled, how can there be a 'loose' bundle at all? These properties-by definition, inessential to the object in question-should play no role in fixing that entity's identity. For this reason, we don't find Simons's approach promising. We also find it somewhat unilluminating since, for each object, the nuclear/loose bundle distinction must be a primitive stipulation of the theory.

We've indicated why we find the nature thesis appealing. But one of our main motivations for adopting it is that it allows us to say something illuminating in response to the grounding problem, raised by Burke (1992), Heller (1990), and Zimmerman (1995), to which we now turn. 


\section{The grounding problem}

Adopting the nature thesis allows us to shed light on the perplexing issue of coincident objects. Here's the puzzle. Bill is glad, given the outside temperature, that the jumper he's wearing is made up of a heavy thread of wool. Let's suppose that the jumper (call it 'Jumpy') and the mass of wool (call it 'Woolly') that makes it up perfectly spatiotemporally coincide. Nevertheless, Jumpy and Woolly are distinct, since Woolly but not Jumpy would survive unravelling, and Jumpy but not Woolly would survive bit-by-bit replacement of some of its material. They differ in their properties, so are distinct entities. Yet how can they be distinct? This is the issue we address in this section.

The most perplexing issue surrounding coincident objects is that they share all their intrinsic material properties and yet differ in other properties (e.g., modal or aesthetic properties). The differences between Jumpy and Woolly 'seem to stand in need of explanation and yet there seems to be no further difference between (Jumpy and Woolly) that is poised to explain, or ground, these differences' (Korman 2011: Sect. 3.1). This is the grounding problem. If Jumpy and Woolly are numerically distinct, then:

there are two objects exactly alike in every empirically discriminable intrinsic respect, one of which has the stamina to withstand pressures and survive changes that the other cannot. Should not two physical objects constructed in precisely the same way out of qualitatively identical parts have the same capacities for survival under similar conditions? (Zimmerman 1995: 87)

Zimmerman's point is a powerful one, for we cannot locate the difference between Jumpy and Woolly in their intrinsic material or spatiotemporal properties. If we held with Wiggins (1967) that Jumpy is distinct from but 'nothing over and above' Woolly (1967: 91), save their modal differences, then there is no way of grounding those differences. Taking Wiggins's line just makes the grounding problem seem that much harder. Johnston (1992) concludes that the differences between Jumpy and Woolly are grounded in facts about our interests in those objects, rather than in their intrinsic properties. They are distinct only because we have such-and-such interests and uses for certain kinds of material object. This is not a conclusion we want to accept: we hold that the distinctness of Jumpy and Woolly is a mind-independent and response-independent fact fully grounded in the identities of Jumpy and Woolly alone.

Essential bundle theory is excellently situated to explain how Jumpy and Woolly differ in their capacities for survival. Consider the functional property being wearable. Both Jumpy and Woolly are wearable, for when one puts Jumpy on, one thereby puts Woolly on too. So being wearable has an instance in the region $r$ common to Jumpy and Woolly. On our analysis of what Jumpy and Woolly are, Jumpy is a bundle of some of the property instances in region $r$, including that instance of being wearable. Woolly too is a bundle of some of the property instances in region $r$, but not including any instance of being wearable. Given that Jumpy and Woolly differ in the constitution of their bundles, they must be distinct 
material objects. So essential bundle theory supports the idea with which we began, that Jumpy and Woolly are distinct. But it also explains how they can coincide: Jumpy and Woolly exactly occupy the same region $r$.

This difference in their respective bundles explains the differences in their persistence conditions. Since Jumpy has an instance of being wearable as a part, it is essentially wearable and so could not survive being unraveled. But Woolly, lacking this part, is inessentially wearable (and also inessentially jumper-shaped, and so on) and hence could survive being unravelled. Jumpy is a jumper because it has all the required properties, such as being wearable, in its bundle. Woolly is not a jumper because its bundle lacks some of these properties. Jumpy and Woolly differ in their modal properties, their persistence conditions, and the sortals they fall under because they differ in their essences, and they differ in their essences simply because they are different bundles of property instances.

According to essential bundle theory, Jumpy's modal properties are grounded in Jumpy's essence. But, one may ask, what grounds Jumpy's essence? What grounds Jumpy's being essentially wearable? Just the fact that the Jumpy-bundle includes an instance of being wearable. The quotidian property being wearable enters into Jumpy's constitution. Nothing more is required to explain why Jumpy is essentially wearable, according to essential bundle theory. Since the Jumpy-bundle is just a mereological sum of property instances, one cannot require a further ground for a given instance's inclusion in that bundle. What grounds that instance's being a part of the Jumpy-bundle is just the Jumpy-bundle itself. In this way, we fully ground Jumpy's and Woolly's relevant modal properties, including their persistence conditions, whilst maintaining their distinctness. This is just the kind of response to the grounding problem we wanted.

It is worth comparing our solution to the grounding problem to Paul's (2006). For Paul, material objects are mereological bundles where (in keeping with traditional bundle theory) those bundles account for property possession simpliciter. She then analyses an object's essential properties in the counterpart-theoretic way, in terms of similarities between bundles. We've already indicated general reasons for dissatisfaction with this approach (Sect. 3). We don't see essential property attribution as being as liberal as the counterpart-theoretic analysis would have it; and we don't think essential properties align with an object's de re modal properties.

There is also a problem specific to Paul's approach. For it to provide a solution to the grounding problem, there must exist coincident bundles which agree in all their intrinsic properties, but which nevertheless differ in the similarity relations they bear to other bundles. Differences in similarity between bundles will not be fully grounded in any of their intrinsic features. It is then a primitive matter which bundles resemble which, on Paul's account. But such facts should not be taken as primitive. Once one fixes the context and the non-similarity facts, one should then be able to say which bundles resemble which (to a given degree, in a given respect). Note that this is the case even if one accepts that similarity relations give a suitable way to analyse essences. (Lewis (1986) holds on Humean grounds that the local facts, which do not include all the facts about similarity, determine all the facts simpliciter, including similarity facts). In short, Paul's analysis locates the ground 
for differences in essence in the wrong kind of fact, and we take that as sufficient reason for preferring our analysis over hers.

In this section, we've set out one of the main motivations for adopting the nature thesis: the natural solution it offers to the grounding problem. We now turn to one of the remaining issues surrounding essential bundle theory, deferred from earlier: what do we say about accidental property possession?

\section{Property possession}

What is it for a material object to possess a property accidentally? How do we fill in the scheme: $x$ is accidentally $F$ if and only if ...? Not in terms of bundlemembership. Having renounced POSSESSION THESIS (Sect. 3), we must come up with an alternative answer.

We are going to offer a distinction between two classes of property and, accordingly, will give two ways to complete the scheme: $x$ is accidentally $F$ if and only if ..., one for each class of property. Consider again our big-picture view: reality is fundamentally no more than a distribution of property instances (including relation instances) over spacetime. At the fundamental level, it's quite likely that those instances are instances of sparse properties: perhaps mass, charge, and geometrical spatiotemporal relations. Those instances (and where they are located) determine the locations of non-fundamental property instances.

However the story goes in detail, the distribution of fundamental property instances determines the distribution of all property instances. Let's introduce the notation ' $[F, r]$ ' for the instance of Fness located throughout spatiotemporal region $r$. For any non-fundamental property $F$, there must be some ground for $[F, r]$. [Being scarlet, $r$ ] will ground [being red, $r$ ], for example; but [being scarlet, $r$ ] will have its own grounds, right down to instances of fundamental properties. (Grounds need not be a single property instance, and need not be confined to $r$ ). The ground explains, ontologically, why $F$ has an instance in $r$ at all. We assume that grounds necessitate what they ground: necessarily, if the grounds for $[F, r]$ exist, then there's an instance of $F$ in $r$. (We take it that necessitation is necessary but insufficient for grounding, since it is not the case that every property grounds each necessary property).

For many properties $F$, the ground for any instance $[F, r]$ is either $r$ itself, or fundamental property instances located wholly within $r$. Examples include existing for exactly 10 years, being spherical and having mass $m$. For such properties $F$, the explanation of why we find an instance of $F$ in $r$ never takes us beyond $r$ itself. To put it another way: given a complete description of the fundamental properties instanced in $r$, plus the grounding-facts about $F$, we could infer whether there's any instance of $F$ in $r$. Let's call such properties region-focused. We'll call all other properties bundle-focused, for reasons that will emerge below. For region-focused properties, the formula for property possession is simple:

(RFP) For region-focused properties $F$, material object $x$ in region $r$ possesses $F$ if and only if $[F, r]$ exists. 
As a consequence, spatiotemporally coincident objects share all of their regionfocused properties.

We have stated this formula in terms of property-possession simpliciter, and not in terms of accidental property possession. A material object may possess some region-focused property $F$ in the way set out by (RFP) and also contain an instance of that property in its bundle. In that case, the object is essentially $F$. So (RFP) does not give a formula for an object's possessing a region-focused property accidentally. Rather, $x$ possesses a region-focused property $F$ accidentally when it possesses $F$ (in accordance with (RFP)) and no instance of $F$ is a part of $x$. So, although coincident objects will share all of their region-focused properties, they may differ in whether they possess those shared properties essentially or accidentally. One might think that an A4 letter has its size essentially, whereas the co-located mass of paper doesn't, for example.

For bundle-focused properties (all the properties that are not region-focused), grounding explanations are typically more complex than they are for region-focused properties. In such cases, an instance $[F, r]$ is not fully grounded by fundamental instances within $r$. One way in which this can happen is when $[F, r]$ depends on various environmental factors external to $r$. Such properties include being financially valuable, being a work of art and being married. For such an $F$, let's call circumstances external to $r F$-suitable when they allow for $F$ to be instanced in $r$ (depending on how $r$ is internally). Circumstances are marriage-suitable, for example, when social conditions are such that a particular sequence of speech acts ('do you ...', 'I do') results in two people being married to one another. What grounds [beingmarried, $r$ ] is then a combination of marriage-suitable circumstances obtaining in $r$ 's spatiotemporal vicinity, in addition to the fundamental instances within $r$ itself.

In this kind of case, the grounding explanation for $[F, r]$ can be factored into two components: (i) the obtaining of $F$-suitable circumstances in some region $r^{\prime}$ causally relevant to $r$; plus (ii) the properties that are instantiated in $r$. $F$-suitable circumstances may be very general, but typically require certain sortal properties to be instantiated in the relevant region. For example, marriage-suitable circumstances require two people to utter certain speech acts. Let's say that person is the key sortal for marriage-suitable circumstances. Our formula for possession of a bundlefocused property $F$ makes use of this notion of $G$ being the key sortal for $F$ suitable circumstances. We don't propose an analysis of this concept here but we trust that the notion is workable in practise.

The property-possession formula for such bundle-focused properties is then:

(BFP) For bundle-focused properties $F$ whose key sortal is $G$, material object $x$ in region $r$ possesses $F$ just in case $[F, r]$ exists and $[G, r]$ is a part of $x$.

This account correctly predicts that it is people, but not the physical lumps which materially constitute them, which possess being married. A physical lump is not essentially a person, even if it is coincident with a person, and so does not contain being a person. (BFP) then tells us that the lump cannot possess being married, even if being married is instantiated in the lump's region. In just the same way, (BFP) 
correctly predicts that certain statues, but not the lumps which materially constitute them, are valuable works of art.

Before moving on, we offer a brief aside on the notion of intrinsic properties. The common idea is that a property is possessed intrinsically when it is possessed independently of how other (wholly distinct) objects are (Kim 1982). But this notion fails as a definition of intrinsic property possession: the property being the only material object satisfies the criterion, yet is a paradigm extrinsic property (Lewis 1983). What's wrong with the independence definition of 'intrinsic' (and its many refinements), from our point of view, is that it is at bottom a modal characterisation of 'intrinsic'. We do not see intrinsicality as a modal phenomenon. In our view, whether a property $F$ is possessed intrinsically depends just on how the relevant instance is in fact grounded.

We offer the following analysis of intrinsic property possession: $x$ (in $r$ ) possesses $F$ intrinsically just in case (i) there exists an instance $[F, r]$, and (ii) the grounding conditions for $[F, r]$ do not involve regions which are not parts of $r$. We can say, derivatively, that $F$ is an intrinsic property (simpliciter) just in case $F$ is possessed intrinsically whenever it is possessed at all. On this analysis, being spherical is intrinsic, whereas being married is not. Moreover, our analysis does not treat being spherical or married as an intrinsic property of spheres, even though the ground for sphere $x$ 's being spherical or married is to be found in $x$ 's region. This is because, even though $x$ is a sphere-or-married because it is a sphere, the grounding conditions for being married, and hence for being spherical or married, appeal to regions beyond $x$ 's. This analysis identifies the intrinsic properties with the regionfocused properties. This seems exactly right: one cannot be intrinsically married, intrinsically valuable, or intrinsically a work of art.

Our analysis of intrinsicality is strictly less inclusive than the independence criterion. If $F$ is intrinsic, by our criterion, then it is possible for something to be $F$ independently of how any wholly distinct thing is, and indeed possible for there to be a lonely $F$. (Just consider the world consisting of nothing but $r$ and the material objects in that region). But properties such as being the only material object are not deemed intrinsic, on our analysis. (They cannot be grounded in any particular region $r$, because it is always possible to duplicate $r$ as part of a larger world containing more material objects). We get the right result here easily, whereas Lewis-style theories cannot (or at least, cannot do so easily).

\section{The problem of relations}

The problem of relations, as faced by traditional bundle theorists, is this. Bundlemembership is supposed to account for a material object's qualitative profile. But part of a material object's qualitative profile will involve the relations it stands in, together with other objects. Bob is 211 miles from the Eiffel tower, as it is from him. So, for traditional bundle theorists who accept PROPERTY POSSESSION ROLE from Sect. 3, Bob's bundle must contain being 211 miles from the Eiffel tower. This would appear to make the Eiffel tower a constituent of the Bob-bundle, violating the 
bundle theorist's maxim that properties and relations are strictly more fundamental than material objects.

It gets worse. Just as Bob's bundle must contain being 211 miles from the Eiffel tower, the Eiffel tower's bundle must contain being 211 miles from Bob. Each bundle is in part relationally constituted by the other. Indeed, since everything bears relations to many things (if not to everything), it will turn out that everything is constituted in part by its relations to many (if not all) other things. If this is not to be blatant metaphysical circularity, it must be a thoroughgoing form of metaphysical holism. Such views may be acceptable, but they are controversial. We do not think any bundle theory which aims to be a very general theory of material objects should be committed to such views.

Essential bundle theory rejects PROPERTY POSSESSION ROLE for bundles, and so bundles need not contain relations in order to stand in them. A bundle will contain a relation (to some entity) only if it is part of the nature of the object to stand in that relation (to that entity). But we certainly want to allow that some material objects are like this. So essential bundle theory does need to explain how relations enter into bundles.

We already have the resources to give an answer. It is relation instances which enter into bundles. A relation instance is very much like a property instance, except that a relation instance is individuated in part by two or more spatiotemporal regions. If we think of relations as universals, then an instance of $n$-place relation $R$ consists of $R$ instantiated by $n$ spatiotemporal regions, $r_{1}, \ldots, r_{n}$, in that order. We'll notate this instance ' $\left[R, r_{1}, \ldots, r_{n}\right]$ '. (Just as in the case of monadic property instances, this does not mean that those regions stand in relation $R$ to one another. Just as we distinguish between a region instantiating a monadic universal and an object possessing it (Sect. 5), so we distinguish between some regions instantiating a relational universal and some objects bearing that relation to one another).

In the case of (monadic) properties, UNIFICATION requires that bundles be unified by some region $r$ (Sect. 2). To extend the idea to relation instances, we will say that a bundle is unified by some region $r$ just in case, for every instance $\left[R, r_{1}, \ldots, r_{n}\right]$ in the bundle and for some $1 \leq i \leq n, r=r_{i}$. (Note that the unification requirement for monadic properties is a special case of this general requirement, where $n=1$ ). Consequently, we update UNIFICATION as follows:

$\left(\right.$ UnIFICATION $^{+}$) Property and relation instances constitute a material object only if there is some spatiotemporal region $r$ such that each such property or relation instance has $r$ as one of its regions.

(Since each property instance is associated with a unique region $r$, UNIFICATION ${ }^{+}$ implies UNIFICATION).

How do we use these relation instances to explain how objects stand in relations? Just as with monadic properties, relations can be either region-focused or bundlefocused. They may be region-focused in one argument-place but bundle-focused in another. The explanation of whether an argument place is region-focused or bundlefocused is very much like the explanation (from Sect. 5) of whether a monadic property is region-focused or bundle-focused. If the fact that region $r_{i}$ instantiates 
$R$ 's $i$ th argument place depends only on $r_{i}$ itself (and on the fundamental properties and relations located wholly within $r_{1}$ ), then $R$ 's $i$ th argument place is regionfocused. Otherwise, it is bundle-focused. To illustrate: being more massive than is region-focused in both argument places, for having mass $m$ is a monadic regionfocused property, whereas being more valuable than is bundle-focused in both argument places.

We associate each bundle-focused argument place with a key sortal (just as we did in Sect. 5 for the case of monadic properties). The dyadic is a parent of is bundle-focused in both argument places, each of which has being a creature as its key sortal. If we have an instance [being a parent of, $r_{1}, r_{2}$ ], and $x$ is a creature in $r_{1}$ and $y$ a creature in $r_{2}$, then $x$ is a parent of $y$. If $x$ has [being a parent of, $r_{1}, r_{2}$ ] as a part, then $x$ is essentially a parent of $y$; otherwise, it is accidental to $x$. Similarly, if $y$ has [being a parent of $\left., r_{1}, r_{2}\right]$ as a part, then $y$ essentially has $x$ as a parent; otherwise, it is accidental to $y$.

The general formula for some material objects bearing a relation to one another is then as follows:

(R) Material objects $x_{1}, \ldots, x_{n}$, whose regions are $r_{1}, \ldots, r_{n}$, bear the $n$-place relation $R$ to one another, in that order, if and only if:

i. the instance $\left[R, r_{1}, \ldots, r_{n}\right]$ exists; and

ii. for each of $R$ 's argument-places, if the $i$ th is bundle-focused and has key sortal $G$, then $\left[G, r_{i}\right]$ is a part of $x_{i}$.

The concepts to which we've appealed here are just those we used in making sense of property possession and essential properties. If we're right that traditional bundle theorists face a serious problem in explaining how objects stand in relations, then it is certainly a positive feature of our story about property possession that is extends easily to relations.

\section{Objections considered}

We will end by considering some worries one might have with the theory. We'll address five worries, concerning 'missing' essential properties; the size of our ontology; weird objects; reference; and causal overdetermination.

Here's the first worry. We identify material objects with suitable bundles (mereological sums) of property instances. That's what material objects are. So, any material object is essentially a bundle of property instances. But we also treat the essential properties of a material object as those with an instance in the bundle. So, any material object should have an instance of the property being a sum of property instances in its bundle. The worry is that this will not be the case.

Our theory solves the issue in a rather neat way. Recall that suitable bundles must be truthmaker closed (Sect. 2). So let's consider what the truthmakers for $\langle x$ is a sum of property instances $\rangle$ might be. It's highly plausible that, quite generally, what makes a sum of $F \mathrm{~s}$ a sum of $F$ s is nothing but those $F$ s. After all, there's nothing to a sum of $F \mathrm{~s}$ but those $F \mathrm{~s}$, and given those $F \mathrm{~s}$, that sum exists. So 
given the property instances that form a bundle $x$, those instances themselves make it the case that $x$ is a bundle of property instances, and hence truthmake $\langle x$ is a sum of property instances $\rangle$. By the closure condition, therefore, any material object $x$ will contain an instance of being a sum of property instances. So if $x$ is a material object, it will (according to our theory) be essentially a sum of property instances, just as the objector required.

Here's the second worry. According to our theory, there exist an awful lot of material objects, far more than in any 'ordinary' ontology. But we do not see this in itself as an objection. It wasn't our intention to deliver an ontology closely matching common sense. We pay a price in quantitative parsimony but, given the theoretical benefits we've outlined above, it's a price we're willing to pay.

Perhaps the objection is not so much that we have too many objects in our ontology, but rather that we accept objects which are a little too weird. Take any bundle of property instances; then, we say, there's a material object which has those properties essentially. It's certainly true that this ontology does not conform to the way our conceptual schemes chop up the world. But consider the alternatives: (i) nature carves the world independently of, but in a way that closely corresponds to, our conceptual schemes; or (ii) nature carves the world in a way that's based on the way our conceptual schemes carve the world. Option (i) is implausible. Our conceptual schemes are carefully tailored to help us get along in the world. But it is anthropocentric hubris to suppose that nature carves reality exactly in the way that would most benefit us.

Option (ii) may be developed as all-out idealism, on which all that exists depends for its nature on our mental life or our concepts. That's very unattractive, given how deeply puzzling the idea of fundamental mental substance is. On an alternative way to develop (ii), the distribution of matter across spacetime is mind-independent, but the carving of that distribution of matter into objects depends on our conceptual scheme. Goswick (2010) discusses an idea along these lines:

We are not, as the standard Realist claims, in the business of trying to ascertain the essential natures of independently existing objects. Rather, we are in the business of creating - given certain distributions of matter in space-timeobjects whose essential natures match our concepts. (Goswick 2010: 443)

The view is that all of reality except the basic distribution of matter depends in some way on our conceptual schemes. But what of the ontological status of concepts themselves? Our conceptual scheme cannot be determined merely by the distribution of matter, on Goswick's view, for otherwise all of reality ends up being determined purely by the distribution of matter. This is precisely the hard realist view Goswick wants to deny. But neither can concepts be completely 'free floating' of the distribution of matter if this view is not to collapse into all-out idealism. We cannot see a clear way out of this problem for Goswick.

In sum, what objects there are does not correspond perfectly to our concepts. So we do not take it as an objection that essential bundle theory posits objects which lie outside our usual conceptual scheme.

There is one form of the 'too many objects' objection which is more serious: the problem of reference. If there are many spatially overlapping objects in any region, 
differing in their nature but agreeing in their material parts, how are our words ever to single out a unique object? An adequate story about reference takes us beyond the scope of this paper but, in short, our response is in two parts. First, context plays a vital role in raising potential referents to salience, from which a referent may be selected. If you say, 'that person over there', or if we are talking about people in general and you point over there, then your words and gesture will single out a bundle that's essentially a person and whose region is roughly over there.

Second, it is often the case that our words fail to single out a unique referent. Consider that nasty-looking cloud overhead. There are many precisely-delineated objects overhead and, plausibly, there is no fact of the matter as to which 'that cloud' refers. It is indeterminate which is the referent of 'that cloud', hence indeterminate where the cloud begins and ends. A similar phenomenon may occur with precisely overlapping objects which differ only in their essences. There may be no fact of the matter as to which of these 'the $F$ ' refers. If so, there will be indeterminacy in what the essence of the $F$ is. This seems to be the right result. There are objects that are (clearly) $F$ for which it seems we do not, and cannot, know whether they are essentially or accidentally $F$. The indeterminacy view can explain why this is so.

Finally, consider the familiar problem of causal overdetermination. Was it the thrown ball, or the mass of rubber that makes it up, that caused the window to break? We have two candidate causes, with equally good claims. But, it is usually said, they can't both be genuine causes, for causal overdetermination is not ubiquitous. This is a problem for any theory which allows for distinct entities to be spatially coincident. Our response is simple. These are cases of causal overdetermination, but overdetermination in such cases is entirely unproblematic. Both the ball and the mass of rubber cause the window to break because the mass of rubber materially constitutes the ball. These material constitution relationships support counterfactuals such as: had the ball not hit the window, neither would the mass of rubber. These cases are very different from the case in which two distinct balls hit the window at once. Such cases are possible but rare: they require very precise conditions. But overdetermination through material constitution is entirely different: it is ubiquitous and unproblematic.

\section{Conclusion}

According to essential bundle theory, material objects are mereological sums of property (and relation) instances which meet certain conditions (Sect. 2). Those instances exhibit both particularity and quality, and hence so do the resulting material objects. The bundle we identify with material object $x$ gives us $x$ 's nature, not $x$ 's full qualitative profile: $x$ (in region $r$ ) is essentially $F$ just in case it has $[F, r]$ as a part (Sect. 3). Given essential bundle theory, we can tell a neat story about how material objects can coincide spatiotemporally and how they may nevertheless differ in their properties (Sect. 4). We can also give a grounding theory of property possession (Sect. 5) which extends naturally to relations (Sect. 6). Essential bundle theory results in a very large ontology containing many weird and wonderful objects, but that's just what we non-idealists should expect anyway (Sect. 7). Reality is plentiful, our concepts selective. 
Open Access This article is distributed under the terms of the Creative Commons Attribution 4.0 International License (http://creativecommons.org/licenses/by/4.0/), which permits unrestricted use, distribution, and reproduction in any medium, provided you give appropriate credit to the original author(s) and the source, provide a link to the Creative Commons license, and indicate if changes were made.

\section{References}

Armstrong, D. M. (2010). Sketch for a systematic metaphysics. Oxford: Oxford University Press. Arntzenius, F. (2014). Space, time, and stuff. Oxford: Oxford University Press.

Barker, S., \& Jago, M. (2014). Monism and material constitution. Pacific Philosophical Quarterly, 95(2), 189-204.

Bennett, J. (1987). Substratum. History of Philosophy Quarterly, 4(2), 197-215.

Black, M. (1952). The identity of indiscernibles. Mind, 61(242), 153-164.

Braddon-Mitchell, D., \& West, C. (2007). Temporal phase pluralism. Philosophy and Phenomenological Research, 62(1), 59-83.

Burke, M. (1992). Copper statues and pieces of copper: A challenge to the standard account. Analysis, $52(1), 12-17$.

Fine, K. (1995). Senses of essence. In R. Sinnott-Armstrong \& Asher (Eds.), Modality, morality, and belief: Essays in honor of Ruth Barcan Marcus (pp. 53-73). Cambridge University Press.

Fine, K., \& Jago, M. (2017). Exact truthmaker logic. unpublished manuscript.

Goswick, D. (2010). Bridging the modal gap. The Journal of Philosophy, 107(8), 432-443.

Hawthorne, J., \& Sider, T. (2002). Locations. Philosophical Topics, 30(1), 53-76.

Heller, M. (1990). The ontology of physical objects: Four-dimensional hunks of matter. Cambridge: Cambridge University Press.

Jago, M. (Ed.). (2016). Essence and the grounding problem. In Reality making (pp. 99-120). Oxford University Press.

Johnston, M. (1992). Constitution is not identity. Mind, 101(401), 89-106.

Kim, J. (1982). Psychophysical supervenience. Philosophical Studies, 41(1), 51-70.

Korman, D. (2011). Ordinary objects. In E. N. Zalta (Ed.), The Stanford encyclopedia of philosophy (2011th ed.). Greensburg: Winter.

Lewis, D. (1983). Extrinsic properties. Philosophical Studies, 44(2), 197-200.

Lewis, D. (1986). On the plurality of worlds. Oxford: Blackwell.

Lewis, D. (2003). Things qua truthmakers. In H. Lillehammer \& G. Rodriguez-Pereyra (Eds.), Real metaphysics (pp. 25-38). London: Routledge.

Lewis, D., \& Rosen, G. (2003). Postscript to 'things qua truthmakers': Negative existentials. In H. Lillehammer \& G. Rodriguez-Pereyra (Eds.), Real Metaphysics (pp. 39-42). London: Routledge.

McDaniel, K. (2001). Tropes and ordinary physical objects. Philosophical Studies, 104(3), 269-290.

Paul, L. A. (2002). Logical parts. Noûs, 36(4), 578-596.

Paul, L. A. (2006). Coincidence as overlap. Nô̂s, 40, 623-659.

Schaffer, J. (2009). Spacetime the one substance. Philosophical Studies, 145(1), 131-148.

Sider, T. (2003). Four-dimensionalism: An ontology of persistence and time. New York: Oxford University Press.

Simons, P. (1994). Particulars in particular clothing. Philosophy and Phenomenological Research, 54(3), 553-575.

Skow, B. (2005). Once upon a spacetime, PhD thesis, New York University.

Stout, G. F. (1921/1971). The nature of universals and propositions. In Landesman, C. (Ed.), The Problem of Universals (pp. 154-166). New York: Basic Books.

Wiggins, D. (1967). Identity and spatio-temporal continuity. Oxford: Blackwell.

Wiggins, D. (1980). Sameness and substance. Oxford: Blackwell.

Williams, D. (1953). On the elements of being. The review of metaphysics, 7, 3-18 and 171-192.

Zimmerman, D. (1995). Theories of masses and problems of constitution. The Philosophical Review, 104(1), 53-110. 